08

\title{
Микроволновой магниторезистивный эффект в сверхрешетке (CoFe/Cu) с отверстиями микронных размеров
}

\author{
(C) А.Б. Ринкевич, ${ }^{1}$ М.А. Миляев, ${ }^{1}$ Е.А. Кузнецов, ${ }^{1,2}$ Д.В. Перов, ${ }^{1}$ А.Ю. Павлова ${ }^{1}$ \\ ${ }^{1}$ Институт фризики металлов им. М.Н. Михеева УрО РАН, \\ 620108 Екатеринбург, Россия \\ ${ }^{2}$ Российский государственный профессионально-педагогический университет, \\ 620012 Екатеринбург, Россия \\ e-mail: rin@imp.uran.ru
}

Поступило в Редакцию 24 ноября 2021 г.

В окончательной редакции 12 января 2022 г.

Принято к публикации 13 января 2022 г.

Изучен микроволновой гигантский магниторезистивный эффект в сверхрешетке $(\mathrm{CoFe} / \mathrm{Cu})$ с отверстиями микронного размера. Выполнены измерения частотных зависимостей коэффициента прохождения, а также зависимостей от магнитного поля микроволновых коэффициентов прохождения и отражения. Измерения выполнены на образцах сверхрешеток без отверстий, с одним отверстием диаметром $6.3 \mu \mathrm{m}$ и с семью отверстиями диаметром $1.7 \mu \mathrm{m}$. Показано, что присутствие отверстия диаметром $6.3 \mu \mathrm{m}$ приводит к значительной частотной зависимости микроволнового гигантского магниторезистивного эффекта. Выполнены магнитные и магниторезистивные измерения образцов сверхрешеток.

Ключевые слова: металлические сверхрешетки, ферромагнитный резонанс, ферромагнитный антирезонанс, микроволновой гигантский магниторезистивный эффект.

DOI: $10.21883 / J T F .2022 .04 .52249 .298-21$

\section{Введение}

Микроволновой гигантский магниторезистивный эффект $(\mu \mathrm{GMR})$ в металлических наноструктурах был обнаружен в работе [1] и впоследствии тщательно исследован [2]. Методика прохождения микроволн была применена к изучению $\mu \mathrm{GMR}$, и было установлено взаимно-однозначное соответствие между гигантским магниторезистивным эффектом (GMR), измеренным на постоянном токе [3-5], и относительными изменениями коэффициента прохождения микроволн [6]. Теория прохождения микроволн через тонкую металлическую пластину развита в [7]. Прохождение микроволн через металлические наноструктуры исследовано для нескольких классов наноструктур. Было установлено, что в многослойных наноструктурах $\mathrm{Co} / \mathrm{Cu}$-эффект $\mu \mathrm{GMR}$ имеет особенно большую величину $[8,9]$. В работе [10] был осуществлен вариант наблюдения высокочастотного GMR при протекании высокочастотных токов перпендикулярно плоскости слоев сверхрешетки. Серия работ была посвящена исследованию $\mu \mathrm{GMR}$ в волне, отраженной от наноструктуры [11-13].

Магноника - это относительно новое направление исследования высокочастотных свойств нанообъектов, в частности, латерально ограниченных пленок и наноструктур [14-20]. Одной из классических задач магноники считается задача о прохождении микроволн через малое по сравнению с длиной волны отверстие в металлической диафрагме $[21,22]$. Для диафрагмы, расположенной в прямоугольном волноводе, эта задача решена теоретически в [23]. В работе [24] эти результаты применены для расчета устройства - направленного ответвителя Бете. Было установлено, что если отверстие заполнено средой с необычным - близким к нулю значением диэлектрической проницаемости, то величина прошедшей мощности резко увеличивается $[25,26]$. Магнитные и микроволновые свойства в системе углублений - „антидотов“ - в пленке пермаллоя исследованы в [27]. Изучение ферромагнитного резонанса (FMR) показало наличие связанных магнитных состояний, локализованных в области отдельного углубления. В [28] исследовано прохождение терагерцового излучения через диафрагму с системой отверстий. Установлено, что на характеристики прошедших волн оказывают влияние два механизма взаимодействия волн с неоднородностями: 1) резонансы, связанные с локальными характеристиками отдельных отверстий и возбуждением волн вблизи них; 2) формирование полей от всей системы отверстий как суммы полей дифракции. Помимо магноники, задачи о пропускании и отражении волн системой периодических и непериодических неоднородностей (отражателей) рассматриваются в сенсорах, основанных на брэгговских решетках (fiber Bragg gratings, FBG) [29]. В магнитных и высокочастотных сенсорах используются также наноструктуры с профилированной (grooved) поверхностью [30]. Наличие профилированной поверхности увеличивает чувствительность сенсора. Во всех перечисленных выше смежных областях исследуется взаимодействие электромагнитных волн с наномасштабными искажениями поверхности или объема пленок, или периодических структур. Однако цели исследования 
и диапазоны частот электромагнитного излучения в каждой области свои.

В настоящей работе ставится задача изучить влияние одного или нескольких отверстий на эффект $\mu \mathrm{GMR}$, наблюдающийся во внешнем магнитном поле. Часть микроволновой мощности будет проходить через отверстия, а остальное через металл сверхрешетки. Если эти части мощности окажутся сопоставимыми по величине, то в результате интерференции изменится частотная зависимость $\mu \mathrm{GMR}$. Так как наличие отверстий приведет к уменьшению $\mu \mathrm{GMR}$, то в качестве объекта исследования разумно выбрать наноструктуры, в которых величина GMR велика. Нами была выбрана система сверхрешеток $(\mathrm{CoFe}) / \mathrm{Cu}$, в которой при оптимальном выборе толщин слоев удается добиться очень высокого магнитосопротивления [31,32]. Другое обстоятельство, благодаря которому выбраны сверхрешетки из системы $(\mathrm{CoFe}) / \mathrm{Cu}$, состоит в том, что в этой системе были зафиксированы спин-волновые резонансы [33]. Поставленная в работе задача отличается от задач FBG, так как нами рассматривается металлическая сверхрешетка, в отличие от диэлектрических структур, изучаемых в системах FBG. Исследуемая нами структура отличается также от наноструктур с профилированной поверхностью, поскольку методом сухого травления в металле наноструктуры были получены сквозные отверстия.

Будет исследовано прохождение микроволн через сверхрешетку системы $(\mathrm{CoFe}) / \mathrm{Cu}$, не имеющую отверстий, и через образцы сверхрешеток с одним и семью отверстиями микронного диаметра. В интервале частот миллиметрового диапазона $26-38 \mathrm{GHz}$ будут изучены частотные зависимости коэффициентов прохождения. На нескольких частотах будет измерен эффект $\mu \mathrm{GMR}$.

\section{1. Образцы и их характеризация}

Сверхрешетки $[(\mathrm{CoFe}) / \mathrm{Cu}]_{n}$ приготовлены методом магнетронного напыления на высоковакуумной прецизионной установке MPS-4000-C6 (ULVAC Inc., Япония). Были приготовлены образцы сверхрешеток составов glass//Ta5.0/PyCr5.0/[C $\left.\mathrm{Co}_{88} \mathrm{Fe}_{12} 1.5 / \mathrm{Cu} 0.95\right]_{24} / \mathrm{PyCr} 3.0$ (образец № 1) и glass//PyCr5.0/[C $\left.\mathrm{Co}_{90} \mathrm{Fe}_{10} 1.5 / \mathrm{Cu} 0.9\right]_{24} / \mathrm{Ta} 3.0$. Число после указания состава слоя дает толщину данного слоя в нанометрах, индекс у квадратных скобок обозначает число пар слоев. Символом Ру обозначен сплав $\mathrm{Fe}_{20} \mathrm{Ni}_{80}$, символом $\mathrm{PyCr}$ - парамагнитный сплав $\left(\mathrm{Fe}_{20} \mathrm{Ni}_{80}\right)_{60} \mathrm{Cr}_{40}$. Толщина спейсера $\mathrm{Cu}$ выбрана таким образом, чтобы образец попадал на первый максимум зависимости величины GMR от толщины спейсера. Образцы выращены на подложке из стекла Corning с толщиной $0.5 \mathrm{~mm}$ для образца № 1 и $0.2 \mathrm{~mm}$ для остальных образцов. Технология роста сверхрешеток $(\mathrm{CoFe}) / \mathrm{Cu}$ описана в работе [32]. Суммарная толщина металла у обоих сверхрешеток составляет соответственно 66.8 и $65.6 \mathrm{~nm}$.
Рентгеновское исследование образцов было выполнено в ЦКП ИФМ УрО РАН на дифрактометре PanAnalytical. Для обеих сверхрешеток на дифрактограмме виден пик от семейства плоскостей (111) ГЦК решетки. Этот пик общий для $\mathrm{Cu}$ и сплава $\mathrm{CoFe}$, так как параметры ГЦК решетки меди и этого сплава очень близки. Вокруг данного пика имеются осцилляции. Вычисленный по угловому положению этих осцилляций период сверхрешеток совпадает с номинальными значениями, полученными по времени и скорости роста. Отсутствие на дифрактограмме остальных характерных пиков ГЦК решетки указывает на то, что в данных образцах сформирована аксиальная текстура $\langle 111\rangle$. Рельеф поверхности образцов исследован методом атомной силовой микроскопии с применением сканирующего зондового микроскопа Solver Next (НТ-МДТ, Зеленоград).
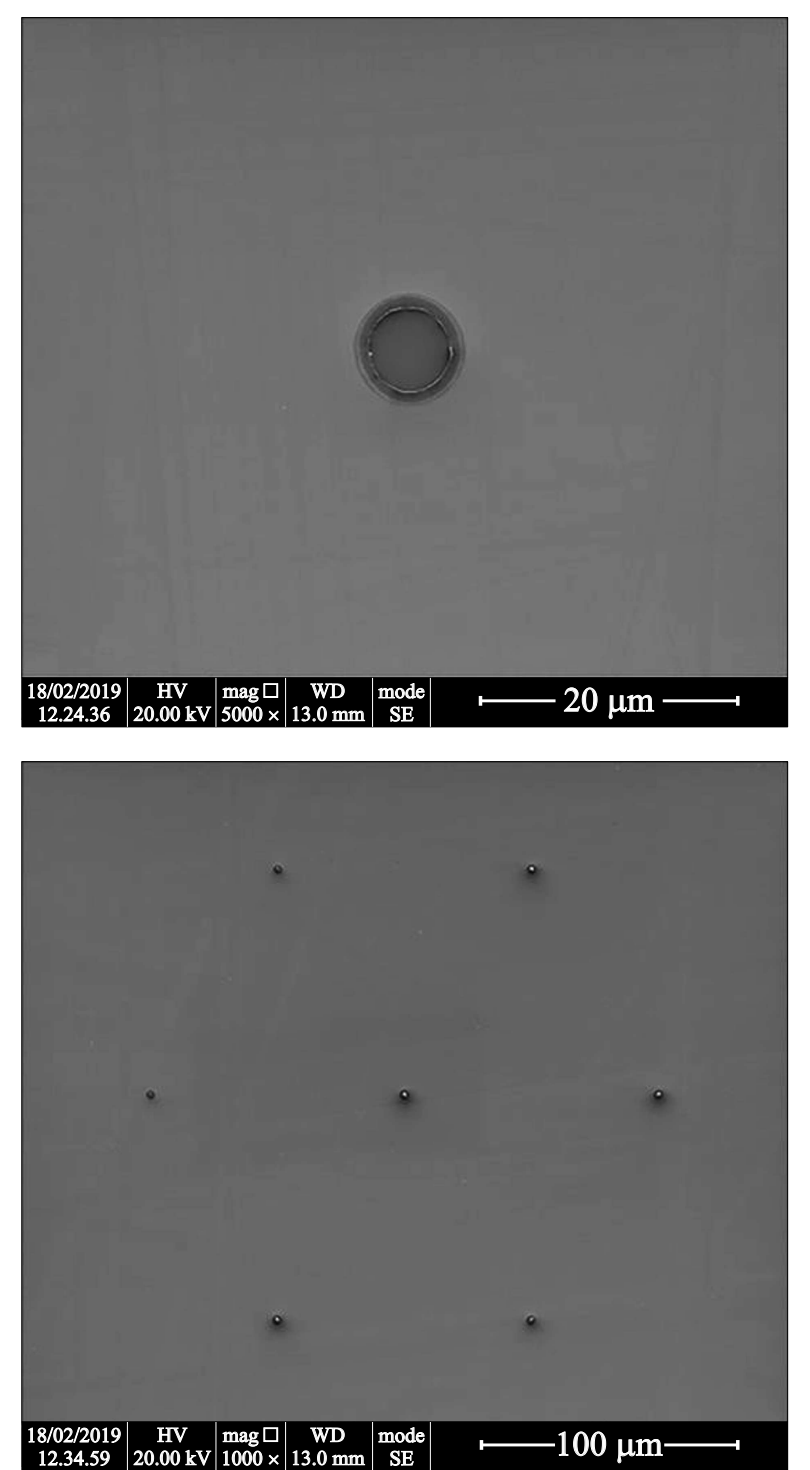

Рис. 1. Электронно-микроскопическое изображение отверстий в образцах сверхрешеток: $a$ - одно отверстие диаметром $6.3 \mu \mathrm{m}$ в образце № $2 ; b-$ семь отверстий диаметром около $1.7 \mu \mathrm{m}$ в образце № 3. Ускоряющее напряжение $20 \mathrm{kV}$. 

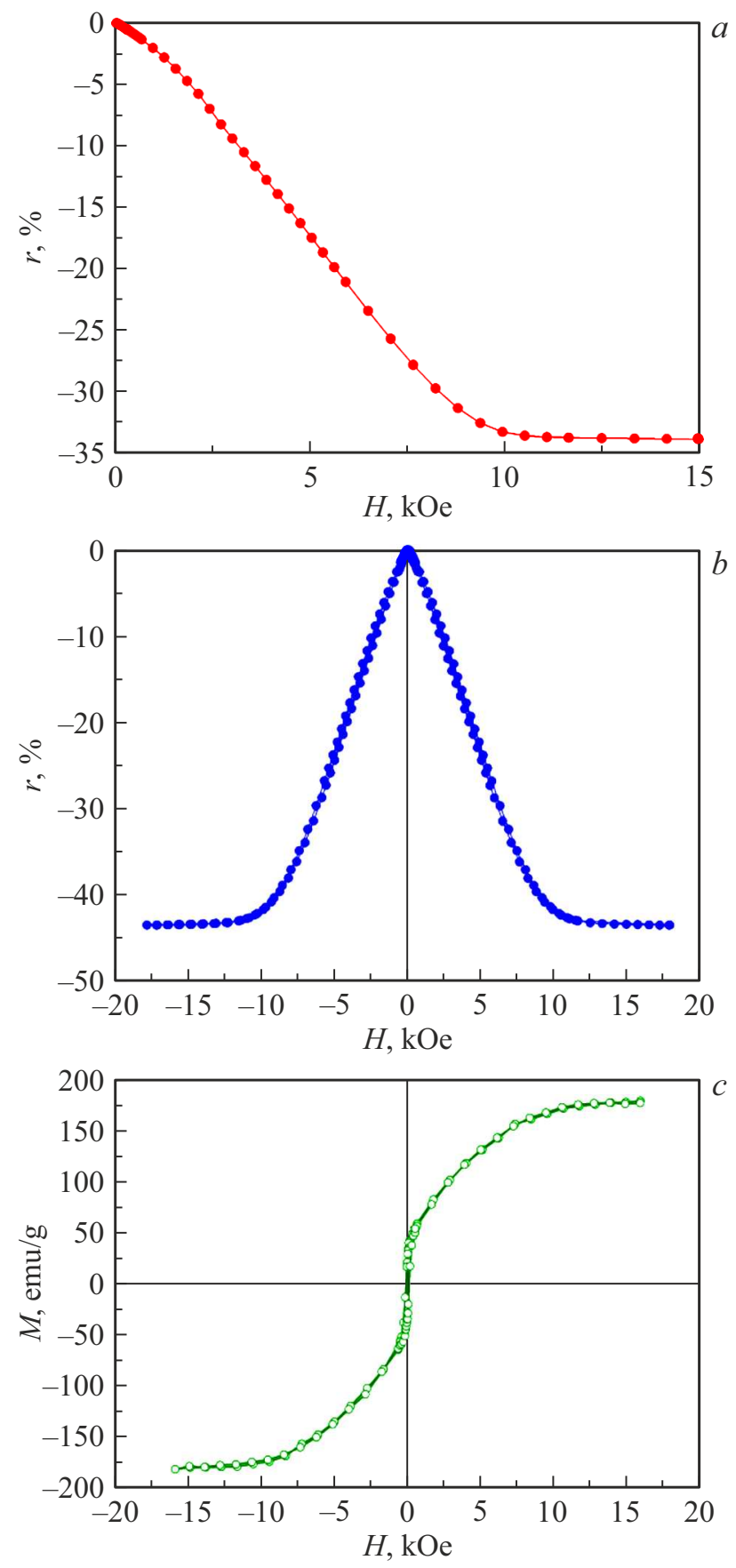

Рис. 2. Магниторезистивные зависимости для образца № $1(a)$ и образцов № 2, № $3(b)$. Петля гистерезиса для образцов № 2, № 3 (c).

Исследование показало, что образцы имеют гладкую поверхность с разностью высот около $3 \mathrm{~nm}$ на поле сканирования $1 \times 1 \mu \mathrm{m}$.

В образцах состава glass $/ / \mathrm{PyCr} 5.0 /\left[\mathrm{Co}_{90} \mathrm{Fe}_{10} 1.5 / \mathrm{Cu} 0.9\right]_{24} / \mathrm{Ta} 3.0$ методом сухого травления были получены сквозные отверстия: одно отверстие диаметром $6.3 \mu \mathrm{m}$ (образец № 2) и семь отверстий диаметром около $1.7 \mu \mathrm{m}$ (образец № 3). Изображения отверстий, полученные мето- дом сканирующей электронной микроскопии, показаны на рис. 1. Отверстия диаметром $1.7 \mu \mathrm{m}$ это минимальные, которые можно было получить с помощью имеющегося оборудования для литографии и сухого травления. Площадь одного такого отверстия очень мала по сравнению с площадью всего образца, и это отверстие не может оказать сколько-нибудь существенного влияния на микроволновые характеристики. Поэтому для образца № 3 выбрана система из 7 отверстий, расположенных на расстоянии, много большем диаметра отверстия. При таком расположении искажения микроволновых полей вблизи каждого из отверстий не распространяются до соседнего отверстия. При этом выбиралось компактное расположение отверстий вблизи центра поперечного сечения волновода, с учетом возможностей литографического оборудования. В образце № 2 выполнено отверстие диаметром $6.3 \mu \mathrm{m}$, что, по оценкам, способно повлиять на прохождение микроволн. Это предположение было подтверждено последующими экспериментами.

В образцах на постоянном токе были измерены магниторезистивные зависимости, которые показаны на рис. $2, a, b$. Для образца № 1 поле насыщения магниторезистивной зависимости составляет примерно $10 \mathrm{kOe}$, величина относительного магнитосопротивления $r=[\rho(H)-\rho(0)] / \rho(0) \cdot 100 \%$, где $\rho(H)-$ электросопротивление в магнитном поле $H$, в насыщении составляет $-34 \%$. Для образцов № 2 и № 3 магниторезистивная зависимость показана на рис. $2, b$. Поле насыщения и для этих образцов составляет $10 \mathrm{kOe}$, a величина максимального магнитосопротивления в насыщении $-43 \%$. Погрешность измерения магнитосопротивления менее $1 \%$. Гистерезис оказывает лишь незначительное влияние на магниторезистивную зависимость, как это видно из рис. $2, b$. Очень большое магнитосопротивление образцов соответствует магнитной структуре слоев, в которой без внешнего магнитного поля реализуется антипараллельное упорядочение моментов соседних слоев. На рис. 2, с показана петля гистерезиса при измерении намагниченности образцов № 2 и № 3. Магнитное насыщение и в этом измерении достигается в полях $\sim 10 \mathrm{kOe}$.

\section{2. Микроволновые измерения}

Микроволновые измерения выполнены при комнатной температуре в интервале частот от 26 до $38 \mathrm{GHz}$. При измерениях образец помещается в оправку, сконструированную так, чтобы предотвратить просачивание электромагнитной энергии у краев образца. Отверстия расположены по центру образцов № 2 и № 3. Схема микроволновых измерений показана на рис. 3. Оправка с образцом 4 помещается в прямоугольный волновод 1 с поперечными размерами $7.2 \times 3.4 \mathrm{~mm}$. Генератор измерителя амплитудно-частотных характеристик возбуждает в волноводе волну, которая падает на образец. Амплитуды отраженной и прошедшей волн измеряются 


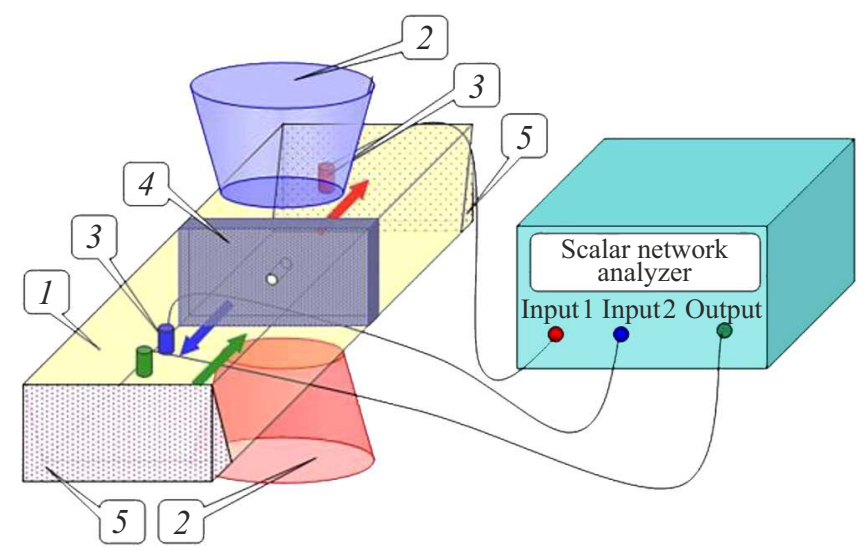

Рис. 3. Схема микроволновых измерений: $1-$ прямоугольный волновод, 2 - электромагнит, 3 - направленные ответвители, 4 - образец, 5 - поглотители.

приемной частью измерителя амплитудно-частотных характеристик с помощью направленных ответвителей 3 . Поглотители 5 предотвращают нежелательные отражения от элементов микроволнового тракта. Внешнее магнитное поле напряженностью до $12 \mathrm{kOe}$ создается электромагнитом 2. Измеряются абсолютные значения модулей коэффициентов прохождения $T$ и отражения $R$ и их относительные изменения в магнитном поле:

$$
\begin{aligned}
d_{m} & =[|T(H)|-|T(0)|] /|T(0)| \cdot 100 \%, \\
r_{m} & =[|R(H)|-|R(0)|] /|R(0)| \cdot 100 \%,
\end{aligned}
$$

где $|T(H)|$ и $|R(H)|$ - значения модулей коэффициентов прохождения и отражения в магнитном поле $H$. Направленные ответвители прошедшей и отраженной волн расположены на расстоянии более $20 \mathrm{~cm}$ от образца, так что высшие типы волн, возникшие на образце как неоднородности в микроволновом тракте, на этом расстоянии затухают, и измерение осуществляется на основном типе волн $\mathrm{TE}_{10}$. Погрешность измерения модуля коэффициента прохождения составляет 5-7\%. Погрешность измерения относительного изменения коэффициента прохождения $d_{m}$ в магнитном поле не более $3 \%$ от измеряемой величины, изменения коэффициента отражения $r_{m}$ не более $10 \%$.

Результаты измерения амплитудно-частотных характеристик коэффициентов прохождения образцов без приложения магнитного поля представлены на рис. 4. Из этого рисунка видно, что присутствие семи отверстий с диаметром $1.7 \mu \mathrm{m}$ лишь незначительно изменяет амплитудно-частотную характеристику. Отверстие диаметром $6.3 \mu \mathrm{m}$ примерно в 1.5 раза увеличивает коэффициент прохождения. Эти результаты могут быть интерпретированы таким образом, что в образце № 3 основная часть микроволновой мощности проходит через металл сверхрешетки, а в образце № 2 мощность микроволн, прошедших через металл и через отверстие, сопоставима.
Перейдем к изучению эффекта $\mu \mathrm{GMR}$ в магнитном поле. Результаты измерения полевой зависимости микроволнового коэффициента прохождения для образца № 1 без отверстий показаны на рис. 5. Микроволновые зависимости в основных чертах подобны зависимости относительного магнитосопротивления, в соответствии c [6]. Однако есть некоторые отличия. Во-первых, величина максимальных микроволновых изменений на 15-25\% больше, чем GMR. Это обстоятельство для системы $(\mathrm{CoFe}) / \mathrm{Cu}$ уже отмечалось ранее в [33]. Выполненный там анализ показал, что это отличие, скорее всего, связано с лишь приближенной пригодностью приближения эффективной среды для многослойной наноструктуры, которое использовано при выводе взаимнооднозначного соответствия в [6]. Второе отличие связано с тем, что существует некоторая частотная зависимость максимальной величины $\mu \mathrm{GMR}$.

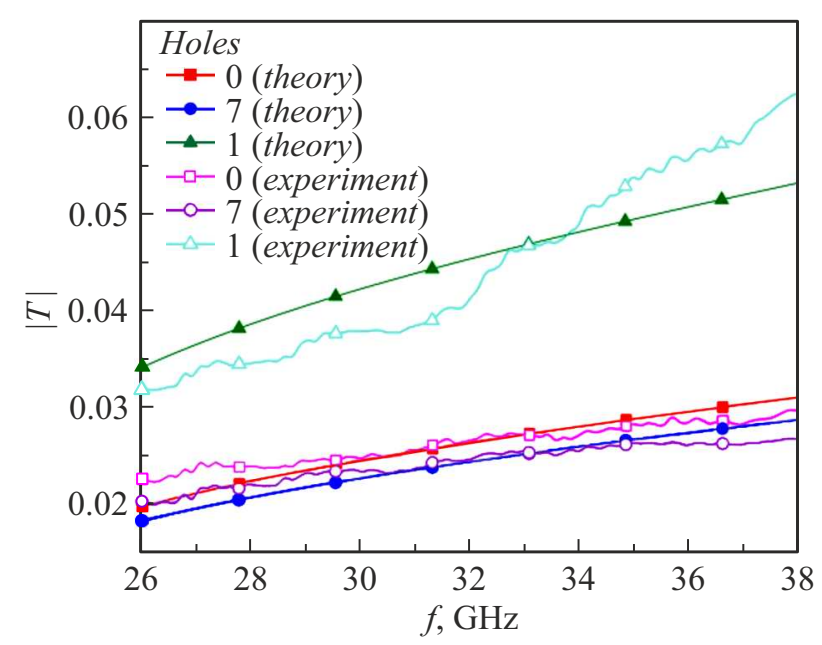

Рис. 4. Амплитудно-частотные характеристики образцов: № 1 без отверстий; № $2-\mathrm{c}$ одним отверстием; № $3-$ с семью отверстиями.

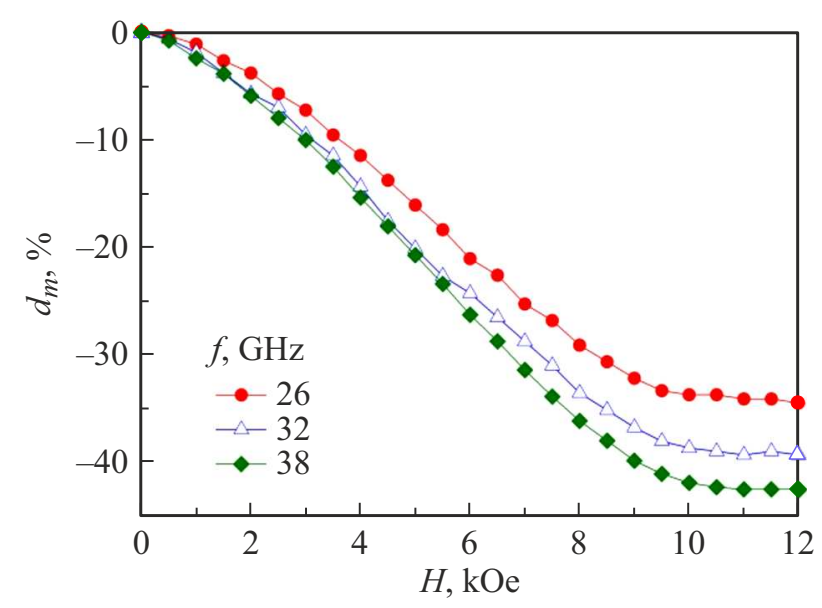

Рис. 5. Зависимость коэффициента прохождения от магнитного поля для образца № 1 без отверстий, измеренная на нескольких частотах миллиметрового диапазона длин волн. 

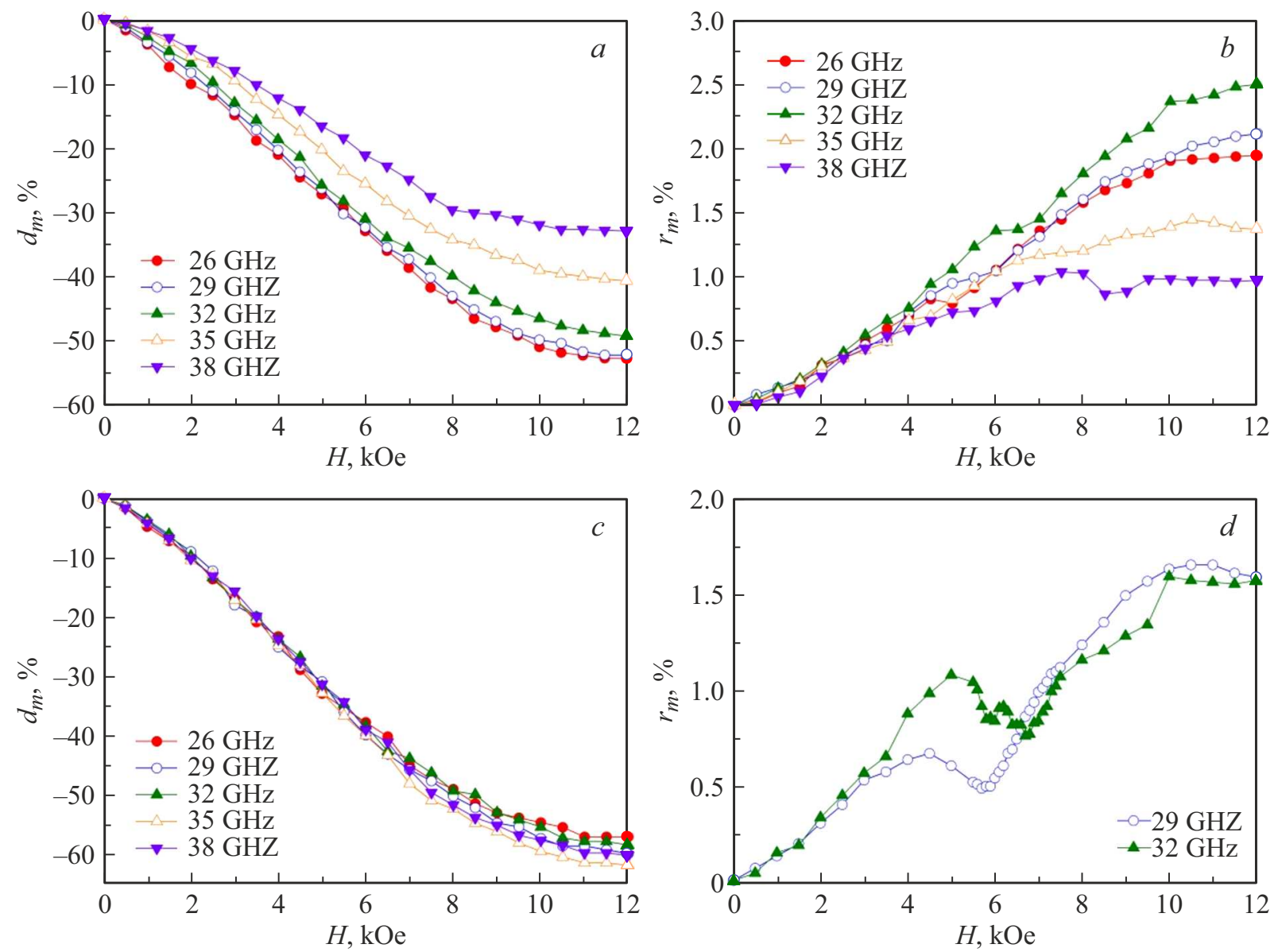

Рис. 6. Зависимости коэффициентов прохождения $(a, c)$ и отражения $(b, d)$ от магнитного поля для образца № 2 с одним отверстием диаметром $6.3 \mu \mathrm{m}(a, b)$ и образца № 3 с семью отверстиями диаметром $1.7 \mu \mathrm{m}(c, d)$, измеренные на нескольких частотах миллиметрового диапазона длин волн.

На рис. 6 показаны зависимости коэффициентов прохождения и отражения от магнитного поля, измеренные в образцах № 2 и № 3 с отверстиями. Сравним результаты измерения коэффициентов прохождения в образцах № 2 и № 3. Как мы установили выше, в измерениях без магнитного поля, в образце № 2 с одним отверстием большего диаметра доля микроволновой мощности, прошедшая через отверстие, сопоставима с мощностью волн, прошедших через металл сверхрешетки. В образце № 3 микроволновая мощность прошедшей волны в основном переносится волной, прошедшей через металл сверхрешетки. В обоих случаях существует качественное подобие зависимостей микроволнового прохождения относительного магнитосопротивления образца. Величина микроволновых изменений несколько больше относительного магнитосопротивления, как и для образца № 1 без отверстий. Однако в образце № 2 с одним отверстием диаметром $6.3 \mu \mathrm{m}$ наблюдается сильно выраженная частотная зависимость максимальных изменений коэффициента прохождения.

Сравним результаты измерения полевой зависимости коэффициентов отражения. Как известно, изменения коэффициента отражения при $\mu \mathrm{GMR}$-эффекте имеют положительный знак, по форме подобны относительному магнитосопротивлению и значительно меньше его по величине [12]. В случае образца № 2 с одним отверстием диаметром $6.3 \mu \mathrm{m}$ мы видим сильную частотную зависимость максимальных микроволновых изменений, как и для коэффициента прохождения. Для образца № 3 с семью отверстиями диаметром $1.7 \mu \mathrm{m}$ на фоне монотонного увеличения коэффициента отражения, вызванного $\mu \mathrm{GMR}$-эффектом, наблюдаются еще максимум, вызванный магнитным антирезонансом (FMAR), и минимум, вызванный поглощением микроволн при выполнении условия FMR [34].

\section{3. Обсуждение результатов}

Коэффициенты прохождения и отражения микроволн можно рассчитать в приближении сплошной среды, в котором многослойная металлическая наноструктура заменяется однородной пластиной такой же толщины с эффективными проводимостью и магнитной проницаемостью. Согласно [35-37], в этом приближении для ко- 
эффициентов прохождения $T$ и отражения $R$ электромагнитной волны можно записать следующие выражения:

$$
\begin{gathered}
T=\frac{2 Z_{m}}{2 Z_{m} \operatorname{ch} k_{m} d+Z \operatorname{sh} k_{m} d}, \\
R=-1+\frac{2 Z_{m} \operatorname{ch} k_{m} d}{2 Z_{m} \operatorname{ch} k_{m} d+Z \operatorname{sh} k_{m} d},
\end{gathered}
$$

где $k_{m}=(1+i) / \delta-$ волновое число в проводящей среде в условиях нормального скин-эффекта, $d-$ толщина металла всей наноструктуры, т.е. суммарная толщина всех металлических слоев. Импеданс наноструктуры равен $Z_{m}=(1+i) \rho / \delta$, где $\rho=\rho(H)$ - удельное электросопротивление наноструктуры, $\delta=\sqrt{2 \rho / \omega \mu \mu_{0}}-$ глубина скин-слоя, $\omega=2 \pi f-$ круговая частота, $\mu-$ относительная динамическая дифференциальная магнитная проницаемость. Заметим, что формулы (1) и (2) получены в предположении [35], что зависимости от времени и пространственной координаты для соответствующих электромагнитных волн, распространяющихся в положительном и отрицательном направлениях вдоль оси, скажем у, имеют вид $\exp \left(i \omega t-k_{m} y\right)$ и $\exp \left(i \omega t+k_{m} y\right)$.

Импеданс волновода, в который помещена наноструктура, на волне типа $\mathrm{TE}_{10}$ рассчитывается по формуле

$$
Z=\sqrt{\frac{\mu_{0} / \varepsilon_{0}}{1-\left(\lambda / \lambda_{c}\right)^{2}}},
$$

где $\lambda=c / f-$ длина волны в вакууме, $\lambda_{c}=2 a-$ критическая длина волны моды $\mathrm{TE}_{10}, a$ - размер большей стенки прямоугольного волновода. В наших экспериментах $a=7.2 \mathrm{~mm}$. Импеданс хорошо проводящей наноструктуры $Z_{m}$ меньше импеданса волновода $Z,\left|Z_{m}\right| \ll Z$. Формулы (1) и (2) будем рассматривать для предельного случая $d \ll \delta$, который реализуется на волнах миллиметрового диапазона. Тогда $2 Z \operatorname{ch} k_{m} d \ll Z \operatorname{sh} k_{m} d$, и коэффициенты прохождения и отражения выразятся формулами

$$
\begin{gathered}
T=\frac{2 Z_{m}}{Z \operatorname{sh} k_{m} d}, \\
R=-1+\frac{2 Z_{m}}{Z} \operatorname{cth} k_{m} d .
\end{gathered}
$$

На рис. 7, $a$ проведено сопоставление зависимостей коэффициента прохождения для образца № 2: измеренной экспериментально, а также рассчитанной по формуле (1), и результатов численного моделирования в программе ANSYS HFSS. Из детального анализа частотных зависимостей коэффициентов прохождения и отражения для образцов № 2 и № 3 получены частотные зависимости эффективной проводимости образцов. Эти зависимости показаны на рис. 7, $b$. Как и следовало ожидать, изменения проводимости сверхрешеток с частотой оказались небольшими.

Было проведено сопоставление зависимостей от магнитного поля коэффициента прохождения микроволн для образца № 3 с семью отверстиями диаметром
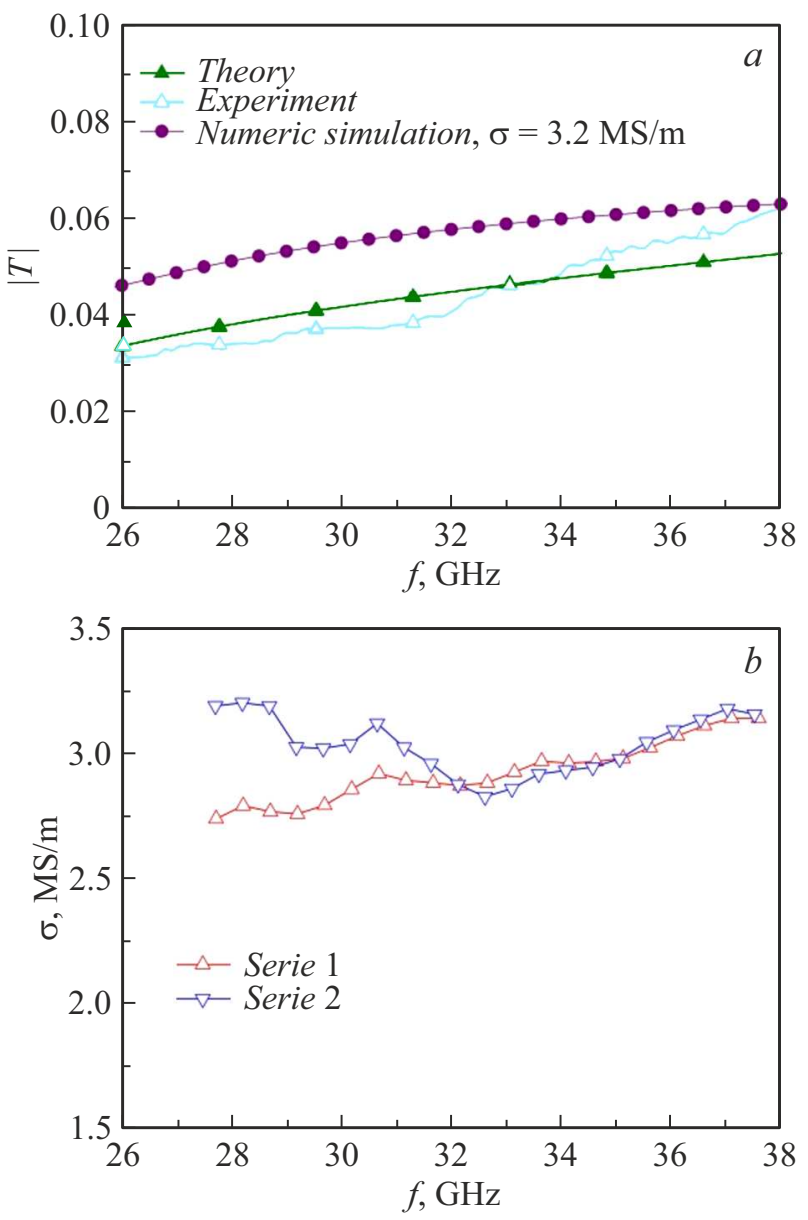

Рис. 7. Сопоставление амплитудно-частотных зависимостей коэффициента прохождения для образца № 2: измеренных, а также рассчитанных по формуле (1) и в программе ANSYS $\operatorname{HFSS}(a)$; частотные зависимости эффективной проводимости образцов № 2 и № $3(b)$.

$1.7 \mu \mathrm{m}$ как рассчитанными по формуле (1), так и измеренными экспериментально (рис. 8). При подобии формы этих зависимостей и знака изменений коэффициента прохождения, следует отметить существенные отличия расчета и эксперимента. Во-первых, величина изменений микроволнового коэффициента в расчете в точности равна GMR-эффекту, измеренному на постоянном токе. У экспериментальных микроволновых зависимостей величина изменений в 1.3-1.4 раза больше. Во-вторых, у расчетных зависимостей практически отсутствует какаялибо частотная зависимость, а у измеренных такая зависимость присутствует.

Отметим приближения, сделанные в расчете, которые могли привести к отличию от результатов эксперимента. В расчете многослойная наноструктура со слоями из разных материалов заменяется пластиной из однородного материала такой же толщины, что и наноструктура с эквивалентными параметрами. Как показано в [33], это допущение только приближенно можно применить к системе $(\mathrm{CoFe}) / \mathrm{Cu}$ с очень большим магнитосопротив- 


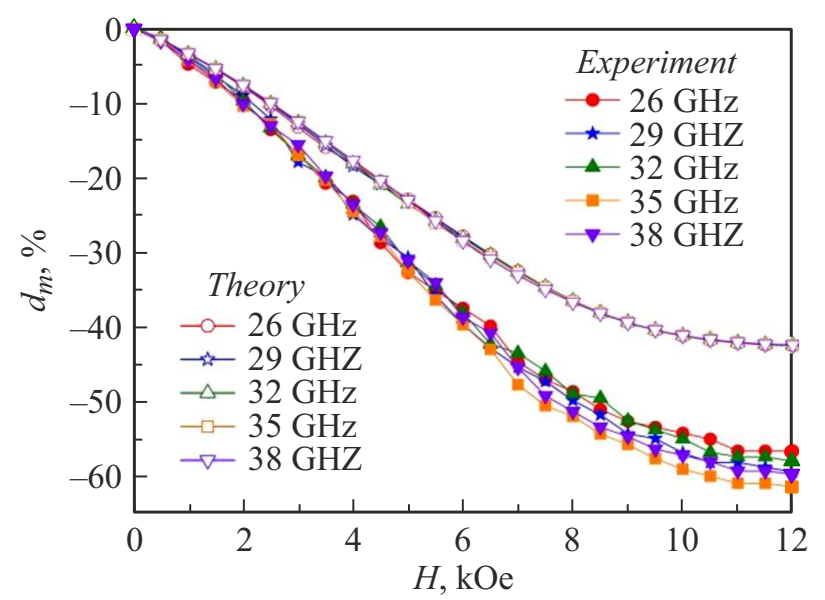

Рис. 8. Зависимости от магнитного поля коэффициента прохождения микроволн для образца № 3 с семью отверстиями диаметром $1.7 \mu \mathrm{m}$, рассчитанными по формуле (1) и измеренными экспериментально.

лением. В то же время для системы $\mathrm{Fe} / \mathrm{Cr}$ с умеренным магнитосопротивлением в $[6,12]$ были получены вполне удовлетворительные результаты.

Второе существенное приближение состоит в том, что влиянием диэлектрической подложки в расчете пренебрегается. Это обосновывается тем обстоятельством, что металл сверхрешетки вносит в микроволновой тракт значительно большие неоднородности, чем диэлектрическая подложка. Кроме того, толщина подложки невелика. Она значительно меньше, чем толщина четверть- или полуволновой пластины.

В результатах расчетов с учетом сделанных предположений наблюдается взаимно-однозначное соответствие между GMR и $\mu \mathrm{GMR}$ при практически полном отсутствии частотной зависимости изменений микроволнового коэффициента прохождения. Эти характерные черты в расчете выполняются в широком частотном интервале от сантиметровых до миллиметровых волн для наноструктур с суммарной толщиной металла от единиц нанометров до десятков микрон. Поэтому есть основания полагать, что зафиксированные выше различия расчета и эксперимента связаны с обсуждаемыми приближениями.

\section{Заключение}

Рассмотрены электродинамические свойства сверхрешеток $(\mathrm{CoFe}) / \mathrm{Cu}$, содержащих отверстия микронного размера. Выполнены измерения амплитудно-частотных характеристик и измерения микроволновых коэффициентов прохождения и отражения в магнитном поле. Измерены магниторезистивные характеристики на постоянном токе. Определена эффективная микроволновая проводимость образцов сверхрешеток.
В сверхрешетках с одним и семью отверстиями исследован микроволновой гигантский магниторезистивный эффект. Установлено, что присутствие отверстия диаметром $6.3 \mu \mathrm{m}$ приводит к значительной частотной зависимости $\mu \mathrm{GMR}$ как в прохождении, так и в отражении микроволн. Величина максимального изменения амплитуды прошедшей волны в интервале частот от 26 до $38 \mathrm{GHz}$ изменяется более чем в 1.5 раза. Присутствие в образце семи отверстий диаметром $1.7 \mu \mathrm{m}$ не приводит к сколько-нибудь заметной дисперсии $\mu \mathrm{GMR}$. В этом образце в отраженном сигнале наблюдались изменения резонансного типа, вызванные FMR и FMAR.

\section{Финансирование работы}

Работа выполнена в рамках тем „Спин“ № АAААА18-118020290104-2 и „Функция“ № АААА-А19119012990095-0. Микроволновые измерения выполнены при поддержке гранта РНФ № 17-12-01002.

\section{Конфликт интересов}

Авторы заявляют, что у них нет конфликта интересов.

\section{Список литературы}

[1] J.J. Krebs, P. Lubitz, A. Chaiken, G.A. Prinz. J. Appl. Phys., 69 (8), 4795 (1991). DOI: 10.1063/1.348232

[2] B.K. Kuanr, A.V. Kuanr, P. Grünberg, G. Nimtz. Phys. Lett. A, 221 (3-4), 245 (1996). DOI: 10.1016/0375-9601(96)00567-1

[3] M.N. Baibich, J.M. Broto, A. Fert, F. Nguyen Van Dau, F. Petroff, P. Eitenne, G. Creuzet, A. Friederich, J. Chazelas. Phys. Rev. Lett., 61 (21), 2472 (1988). DOI: $10.1103 /$ PhysRevLett.61.2472

[4] G. Binasch, P. Grünberg, F. Saurenbach, W. Zinn. Phys. Rev. B, 39 (7), 4828 (1989). DOI: 10.1103/PhysRevB.39.4828

[5] P. Bruno. Phys. Rev. B, 52 (1), 411 (1995). DOI: $10.1103 /$ PhysRevB.52.411

[6] V.V. Ustinov, A.B. Rinkevich, L.N. Romashev, V.I. Minin. JMMM, 177-181, 1205 (1998). DOI: $10.1016 / \mathrm{S} 0304-8853(97) 00279-5$

[7] Е.М. Коган, Е.А. Туров, В.В. Устинов. ФММ, 53 (2), 223 (1982). [E.M. Kogan, E.A. Turov, V.V. Ustinov. Phys. Met. Metallogr., 53 (2), 223 (1982).]

[8] T. Rausch, T. Szczurek, M. Schlesinger. J. Appl. Phys., 85 (1), 314 (1999). DOI: 10.1063/1.369448

[9] D.P. Belozorov, V.N. Derkach, S.V. Nedukh, A.G. Ravlik, S.T. Roschenko, I.G. Shipkova, S.I. Tarapov, F. Yildiz. Int. J. Infrared Milli. Waves., 22 (11), 1669 (2001). DOI: $10.1023 / \mathrm{A}: 1015060515794$

[10] V.V. Ustinov, A.B. Rinkevich, L.N. Romashev. JMMM, 198-199, 82 (1999). DOI: 10.1016/S0304-8853(98)00631-3

[11] Z. Frait, P. Šturc, K. Temst, Y. Bruynseraede, I. Vávra. Solid State Commun., 112 (10), 569 (1999). DOI: $10.1016 / \mathrm{S} 0038-1098(99) 00392-0$

[12] В.В. Устинов, А.Б. Ринкевич, Л.Н. Ромашев, Е.А. Кузнецов. ЖТФ, $79(8), 71$ (2009). [V.V. Ustinov, A.B. Rinkevich, L.N. Romashev, E.A. Kuznetsov. Tech. Phys., 54 (8), 1156 (2009). DOI: 10.1134/S1063784209080106] 
[13] D.E. Endean, J.N. Heyman, S. Maat, E. Dan Dahlberg. Phys. Rev. B, 84 (21), 212405 (2011). DOI: $10.1103 /$ PhysRevB.84.212405

[14] A.V. Chumak, V.I. Vasyuchka, A.A. Serga, B. Hillebrands. Nat. Phys., 11 (6), 453 (2015). DOI: 10.1038/nphys3347

[15] B. Divinskiy, V.E. Demidov, S.O. Demokritov, A.B. Rinkevich, S. Urazhdin. Appl. Phys. Lett., 109 (25), 252401 (2016). DOI: $10.1063 / 1.4972244$

[16] С.А. Никитов, Д.В. Калябин, И.В. Лисенков, А.Н. Славин, Ю.Н. Барабаненков, С.А. Осокин, А.В. Садовников, Е.Н. Бегинин, М.А. Морозова, Ю.П. Шараевский, Ю.А. Филимонов, Ю.В. Хивинцев, С.Л. Высоцкий, В.К. Сахаров, Е.С. Павлов. УФН, $\mathbf{1 8 5}(10), 1099$ (2015). DOI: 10.3367/UFNr.0185.201510m.1099 [S.A. Nikitov, D.V. Kalyabin, I.V. Lisenkov, A.N. Slavin, Yu.N. Barabanenkov, S.A. Osokin, A.V. Sadovnikov, E.N. Beginin, M.A. Morozova, Yu.P. Sharaevsky, Yu.A. Filimonov, Yu.V. Khivintsev, S.L. Vysotsky, V.K. Sakharov, E.S. Pavlov. Phys. Usp., 58(10), 1002 (2015). DOI: 10.3367/UFNe.0185.201510m.1099]

[17] А. Ферт. УФН, 178 (12), 1336 (2008). DOI: 10.3367/UFNr. 0178.200812f.1336 [A. Fert. Phys. Usp., 51 (12), 1336 (2008). DOI: $10.3367 /$ UFNr.0178.200812f.1336]

[18] M. Farle, T. Silva, G. Woltersdorf. In: Magnetic Nanostructures, Spin Dynamics and Spin Transport, ed. by H. Zabel, M. Farle. (Springer-Verlag, Berlin, Heidelberg, 2013), p. 37. DOI: $10.1007 / 978-3-642-32042-2$

[19] X. Zhang, W. Butler. In: Handbook of Spintronics, ed. by Y. Xu, D.D. Awschalom, J. Nitta. (Springer, Dordrecht, Heidelberg, NY., London, 2016), p. 3. DOI: 10.1007/978-94-007-6892-5

[20] Ultrathin Magnetic Structures, ed. by B. Heinrich, J.A.C. Bland. (Springer, Berlin Heidelberg, NY., 2005), v. IV. DOI: $10.1007 / \mathrm{b} 138704$

[21] R.E. Collin. Field Theory of Guided Waves (WileyInterscience-IEEE, NY., Chichester, Weinheim, Brisbane, Singapore, Toronto, 1991)

[22] M. Skorobogatiy. Nanostructured and Subwavelength Waveguides: Fundamentals and Applications (John Wiley \& Sons, Chichester, 2012)

[23] N. Marinescu. Phys. Rev. E, 56 (2), 2166 (1997). DOI: 10.1103/PhysRevE.56.2166

[24] N. Marinescu. Phys. Rev. E, 54 (3), 2931 (1996). DOI: 10.1103/PhysRevE.54.2931

[25] M.G. Silveirinha, N. Engheta. Phys. Rev. Lett., 97 (15), 157403 (2006). DOI: 10.1103/PhysRevLett.97.157403

[26] S.A. Maier. Plasmonics: Fundamentals and Applications (Springer Science + Business Media LLC, NY., 2007), DOI: $10.1007 / 0-387-37825-1$

[27] A.S. Silva, A. Hierro-Rodriguez, S.A. Bunyaev, G.N. Kakazei, O.V. Dobrovolskiy, C. Redondo, R. Morales, H. Crespo, D. Navas. AIP Advances, 9(3), 035136 (2019). DOI: $10.1063 / 1.5080111$

[28] V. Lomakin, S. Li, E. Michielssen. Microw. Opt. Technol. Lett., 49 (7), 1554 (2007). DOI: 10.1002/mop.22484

[29] A. Othonos, K. Kalli. Fiber Bragg Gratings: Fundamentals and Applications in Telecommunications and Sensing (Artech House, Norwood, 1999)

[30] Y. Mu, P. Li, Y. Wen. IEEE Sens. J., 21 (20), 22623 (2021). DOI: 10.1109/JSEN.2021.3110870

[31] W. Kuch, A.C. Marley, S.S.P. Parkin. J. Appl. Phys., 83 (9), 4709 (1998). DOI: 10.1063/1.367259
[32] М.А. Миляев, Л.И. Наумова, В.В. Устинов. ФММ, 119 (12), 1224 (2018). DOI: 10.1134/S0015323018120136 [M.A. Milyaev, L.I. Naumova, V.V. Ustinov. Phys. Met. Metallogr., 119 (12), 1162 (2018). DOI: $10.1134 / \mathrm{S} 0031918 X 1812013 \mathrm{X}]$

[33] В.В. Устинов, А.Б. Ринкевич, И.Г. Важенина, M.А. Миляев. ЖЭТФ, $158(1), \quad 139 \quad$ (2020). DOI: $\quad 10.31857 /$ S0044451020070135 [V.V. Ustinov, A.B. Rinkevich, I.G. Vazhenina, M.A. Milyaev. JETP, 131 (1), 139 (2020). DOI: 10.1134/S1063776120070171]

[34] В.В. Устинов, А.Б. Ринкевич, Д.В. Перов, А.М. Бурханов, М.И. Самойлович, С.М. Клещева, Е.А. Кузнецов. ЖТФ, 83 (4), 104 (2013). [V.V. Ustinov, A.B. Rinkevich, D.V. Perov, A.M. Burkhanov, M.I. Samoylovich, S.M. Kleshcheva, E.A. Kuznetsov. 58 (4), 568 (2013). DOI: $10.1134 / \mathrm{S} 1063784213040257]$

[35] Н.А. Семенов. Техническая электродинамика (Связь, М., 1972)

[36] Л.М. Бреховских. Волны в слоистых средах (Наука, M., 1973) [L.M. Brekhovskikh. Waves in Layered Media (Academic Press, London, 1980)]

[37] L.F. Chen, C.K. Ong, C.P. Neo, V.V. Varadan, V.K. Varadan. Microwave Electronics: Measurement and Materials Characterization (John Wiley \& Sons, Hoboken, 2004), DOI: $10.1002 / 0470020466$ 